\title{
Uretero-duodenal Fistula as a Result from Ureteropelvic Junction Obstruction Correction: Case Report
}

Teeranop Choorit, M.D., Worapat Attawettayanon, M.D., Virote Chalieopanyarwong, M.D.

Division of Urology, Department of Surgery, Faculty of Medicine, Prince of Songkla University, Hat Yai, Songkhla 90110, Thailand. Received 8 May $2020 \bullet$ Revised 3 July 2020 • Accepted 11 July 2020 • Published online 11 November 2020

\begin{abstract}
:
Uretero-duodenal fistula is an uncommon complication in urology. The cause of fistula have been reported as ureteral calculi, iatrogenic injury, trauma and malignancy. The gold standard treatment of uretero-duodenal fistula remains controversial. The most common management is nephrectomy and primary closure of fistula. We report a case of 67-yearold man with uretero-duodenal fistula after laparoscopic correction of ureteropelvic junction obstruction. In the era of minimal invasive management, we decided to use endoscopic and conservative treatment. The definitive treatment will be reconsidered if conservative treatment fails. We will discuss the steps of management and follow-up for this patient.
\end{abstract}

Keywords: fistula, intestinal fistula, ureteropelvic junction obstruction, urinary fistula

Contact: Virote Chalieopanyarwong, M.D.

Division of Urology, Department of Surgery, Faculty of Medicine,

Prince of Songkla University, Hat Yai, Songkhla 90110, Thailand.

E-mail: vchalieopanyarwong@gmail.com
J Health Sci Med Res 2021;39(3):245-249 doi: 10.31584 /jhsmr.2020772 www.jhsmr.org

(ㄷ 2020 JHSMR. Hosting by Prince of Songkla University. All rights reserved.

This is an open access article under the CC BY-NC-ND license

(http://www.jhsmr.org/index.php/jhsmr/about/editorialPolicies\#openAccessPolicy). 


\section{Introduction}

Fistula between kidney and duodenum are an extremely rare. The main cause of this condition are inflammatory diseases such as urolithiasis, pyogenic infection, inflammatory bowel disease and trauma from surgery or intervention procedures. The presentation of uretero-duodenal fistula include upper and lower urinary tract symptoms. The diagnostic test of choice in more recent studies were antegrade pyelogram (AP), esophagogastroduodenoscopy (EGD) and contrast enhanced computer tomography (CT). Recent literature shows the most successful treatment of this situation is nephrectomy and duodenal closure. We would like to report a case of 67-year-old man who was diagnosed with ureteroduodenal fistula whom presented with upper urinary tract symptom after surgical procedure. The initial management was conservative treatment with endoscopic ligation which eventually led to surgical nephrectomy.

\section{Case Report}

A 67-year-old male presented with right flank pain and fever for 10 days after removal of ureteral stent from laparoscopic pyeloplasty procedure. He did not have gastrointestinal symptoms or dysuria and had normal character of urine. Seven years ago, he suffered from right renal pelvis stone; therefore, he underwent open right pyelolithotomy with stone removal at a local hospital. Several months before this illness, he had colicky pain on right-side of abdomen, passed turbid urine, he denied history of lower urinary tract symptoms and passing stones. Laboratory examination revealed pyuria and microscopic hematuria on urinary analysis. His serum creatinine was 1.12 milligrams per deciliter.

Computerized tomography scan of the kidneys, ureters and bladder showed multiple right renal pelvic stones, size about 0.4-1.1 centimeter. Disproportional narrowing of right ureteropelvic junction (UPJ) without demonstrated enhancing lesion. Renal scan differential function Left: Right was 72.0\%:28.0\%, $\mathrm{T}_{1 / 2}$ in left and right was 13.7 and >20 minutes, respectively. The diagnosis was right renal calculi with right UPJ obstruction. Hence, he took laparoscopic right pyelolithotomy with pyeloplasty with Foley $\mathrm{Y}-\mathrm{V}$ plasty. The operative finding was severe adhesion around the right upper ureter and UPJ area. In this patient, we removed a ureteral stent 4 weeks after pyeloplasty. After removal of ureteral stent for 10 days, patient visited emergency department due to high grade fever. His body temperature was $38.3{ }^{\circ} \mathrm{C}$, Blood pressure was 130/80 millimeter $(\mathrm{mm})$ of mercury and heart rate was 110 beats per minute. Physical examination found only right costovertebral angle tenderness. Laboratory studies showed numerous white blood cell on urine analysis and leukocytosis. The patient was diagnosed acute complicated pyelonephritis. After admission patient received intravenous fluid and intravenous antibiotic, but clinical condition did not improve within 24 hours, so patient was then sent for further investigation. CT scan was performed and the result was multiseptated thick rim enhancing mass abutting right psoas muscle, duodenum and ascending colon with right hydronephrosis (Figure 1). We intended to perform retrograde pyelography (RP) and ureteral stent insertion. In addition, RP finding was performed and revealed passage of contrast media from right ureter to the duodenum (Figure 2). We were unable to cannulate guide wire to renal pelvic area, so the operation was terminated after diagnosis of uretero-duodenal fistula. A right percutaneous nephrostomy (PCN) was performed and after that we prescribed total parenteral nutrition and intravenous antibiotics and sent the patient for EGD and ligation fistula tract with the aim of preserving kidney function and avoiding a major operation. After clinical condition was stable, EGD was performed by a gastroenterologist, they discovered the fistula opening $8 \mathrm{~mm}$ at $2^{\text {nd }}$ part of duodenal (Figure 3) and over endoscopic clipping 
successful (Figure 4). After appropriate antibiotics with endoscopic clipping, the patient was maintained on right PCN. His fever, flank pain and overall clinical was improved. He was discharged from service with $\mathrm{PCN}$ at day 15 after admission. During the conservative treatment, the patient had soft diet with low fiber as the colorectal surgeon suggested. Unfortunately, he was re-admitted at a local hospital for recurrent right pyelonephritis 2 times within a month. The patient was sent for AP and the result revealed a communication between proximal ureter and duodenum. The patient decided to undergo nephrectomy with closure of the fistula. The operation was performed at day 50 after endoscopic treatment, intraoperative finding was dense fibrosis which appeared as a band at proximal ureter and $2^{\text {nd }}$ part of duodenum. The bowel defect was repaired and right nephrectomy was performed. On followup, the patient had no clinical flank pain nor abdominal discomfort and was free from urinary tract infection.

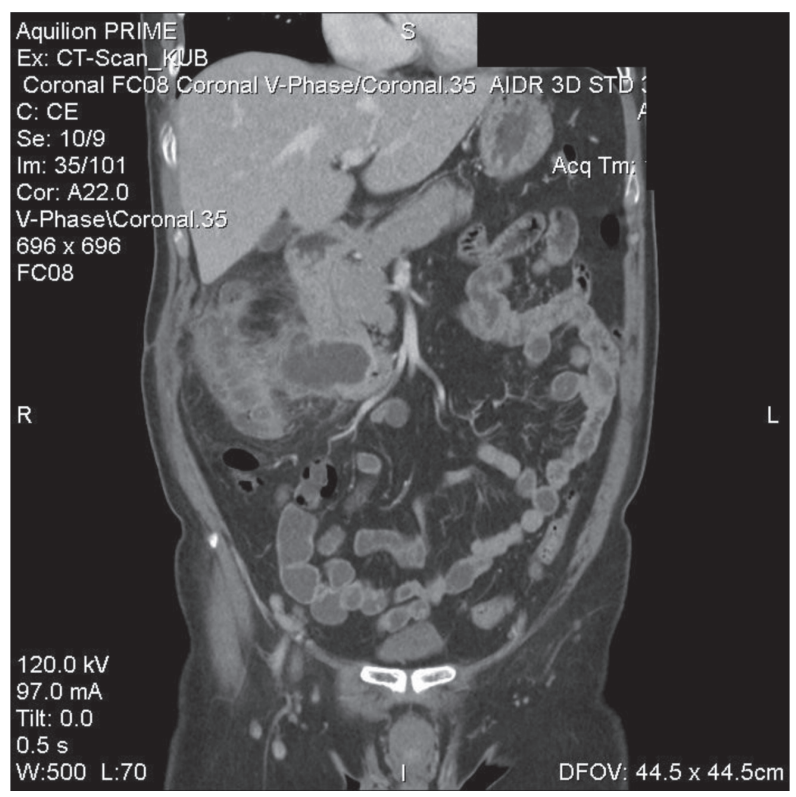

Figure 1 Computerized tomography scan showed multiseptated thick rim enhancing mass abutting duodenum and ascending colon

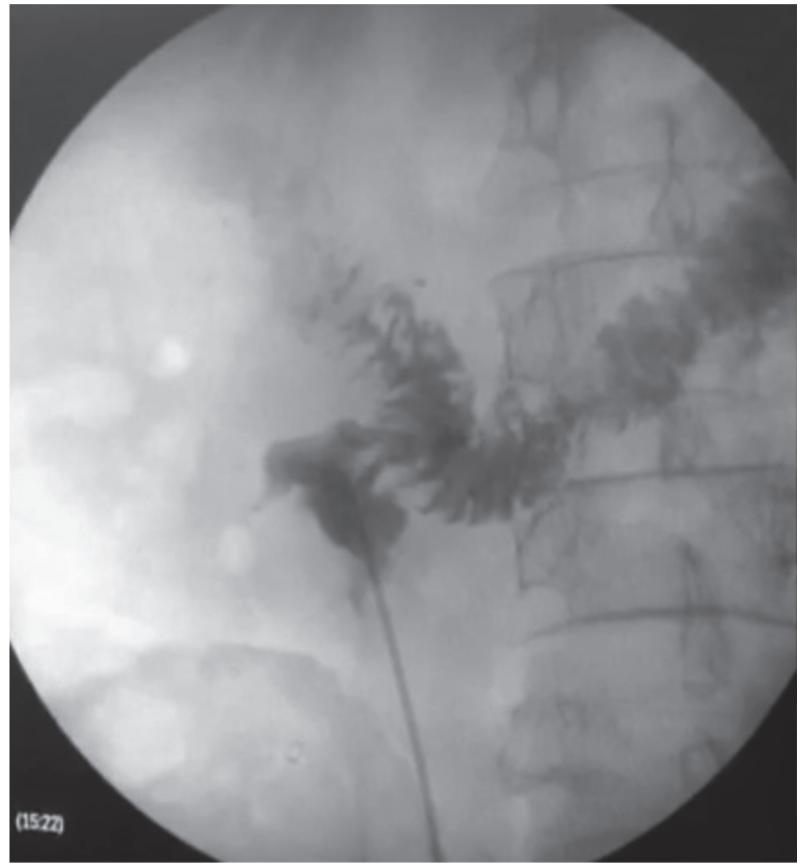

Figure 2 Retrograde pyelography finding was performed and revealed passage of contrast media from right ureter to the duodenum

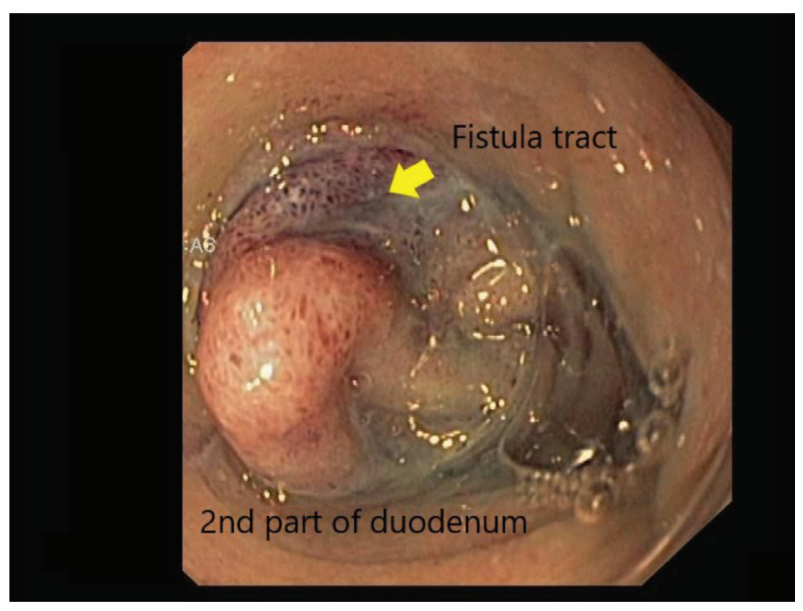

Figure 3 Esophago-gastroduodenoscopy was performed and discovered the fistula at $2^{\text {nd }}$ part of duodenal. The arrow is methylene blue stained mucosa from injection via right percutaneous nephrostomy that suspected fistula tract 


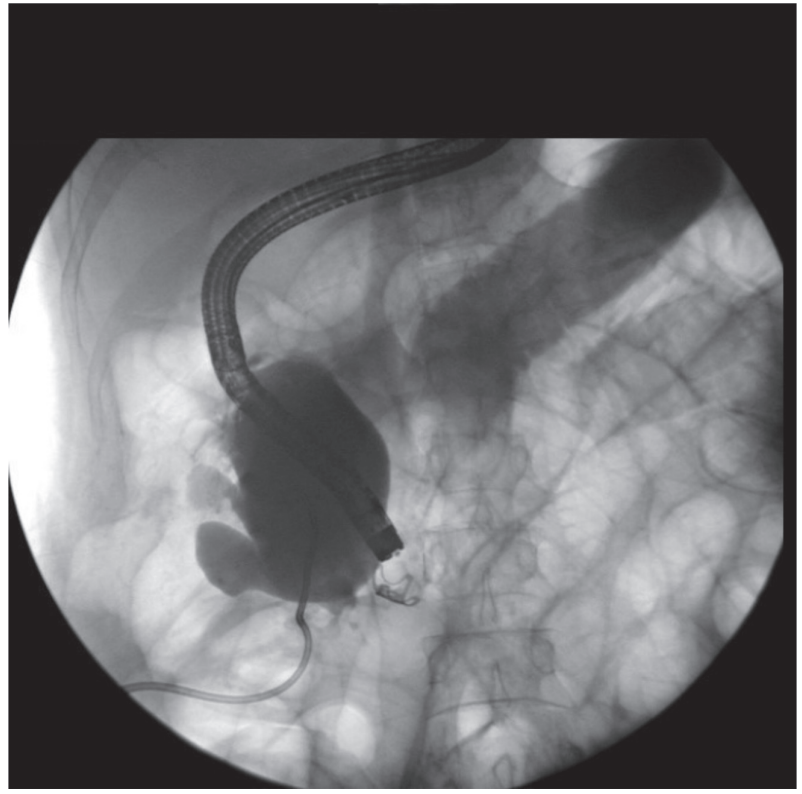

Figure 4 Successful endoscopic clipping for ureteroduodenal fistula

\section{Discussion}

Uretero-duodenal fistula is an unusual condition. The first case was reported in 1918 by David. ${ }^{1}$ The second was not reported until 1968. The cause of both cases were chronic renal infection, successfully treated with nephrectomy and closure of duodenal defect. ${ }^{2}$ Other causes of fistulas have been reported as secondary to ureteral calculi, roundworm infection, iatrogenic injury, trauma, malignancy and idiopathy. ${ }^{3-5}$ Common presentations of this condition were flank pain, fever, pyuria, pneumaturia and upper gastrointestinal tract symptoms such as anorexia, nausea and vomiting. Recurrent pyelonephritis and lower urinary tract infection are also common presentations of this condition. The diagnosis of uretero-duodenal fistula generally required confirmation of connection between from urinary tract and gastrointestinal tract. ${ }^{6}$ Recent evidence mentioned RP, AP or contrast enhanced CT as the diagnostics of choice. ${ }^{6-8}$ The ideal treatment of ureteroduodenal fistula remains controversial. In generally, tradi- tional management is nephrectomy and primary closure of fistula. Literature review by Rodney et al. ${ }^{7}$ stated nephrectomy and duodenal closure were performed in 21 cases of a total of 28 cases, the remaining patients were treated without surgery. They recommend performing operations in patients with long term primary kidney disease. However, there are several recent reports of successful conservative treatment. ${ }^{9,10}$ The treatment includes intravenous antibiotic, urinary diversion with percutaneous drainage or internal ureteral stent and endoscopic treatment with ligation. ${ }^{9}$

In our case, the cause of the fistula is suspected to come from iatrogenic duodenal injury and intra operative detection is very challenging from a laparoscopic view if it had no obvious point of injury. The point to learn in this case is the rare complication of laparoscopic pyeloplasty with UPJ stone that may have severe adhesion to surrounding organs such as duodenum. The prevention needs meticulous dissection and high index of suspicion of duodenal injury in the perioperative phase. We tried to preserve his renal function and avoid major surgery. The predictive factors to consider prior to making a clinical decision were low output fistula and resolved clinical infection during admission. After review of literature, we found previous reports of successful conservative management ${ }^{1,12}$, thus conservative treatment was chosen. Initially, we treated with EGD with clipping and urinary diversion with PCN. Unfortunately, the treatment failed and the patient had recurrent right pyelonephritis 2 times within a month postoperatively. The main cause of unsuccessful treatment may have been from the fistula tract being too large for this intervention, poor quality of tissue and ongoing inflammation process.

\section{Conclusion}

In summary, uretero-duodenal fistula are serious complications after urological procedure. Especially, in situations where there is dense fibrosis around the ureter of renal pelvis. This should also be kept in mind in 
all cases. Treatment should be depend on primary kidney disease and patient's status. If feasible, initial treatment should be conservative. In contrast, if the primary disease process is apparent in the kidney for a long time and conservative treatment fails, nephrectomy and primary closure of fistula should be considered.

\section{References}

1. David EG. Duodenoureteral fistula of spontaneous origin. JAMA 1918;70:376.

2. Robert BJ, Giblin JG, Tehan TJ, Lynch JH. Ureteroduodenal fistula. Urology 1996;48:301-3.

3. Ginsberg DA, Stein JP, Grossfeld GD, Tarter T, Skinner DG Traumatic pyeloduodenal fistula: a case report and review of the literature. Urology 1996;47:588-91.

4. Ross JM, Tanna DD. Pyeloduodenal fistula. J R Coll Surg Edinb 1974;19:51-3.

5. Kondapi D, Tambe V, Hegazy H. Pyeloduodenal fistula as a result of pyonephrosis. Urol Case Rep 2018;30:36-7.
6. Kobayashi T, Casablanca NM, Harrington M. Pyeloduodenal fistula diagnosed with technetium-99m scintigraphy and managed with a conservative strategy. BMJ Case Rep 2018. doi:10.1136/bcr-2017-223425.

7. Rodney K, Maxted WC, Pahira JJ. Pyeloduodenal fistula. Urology 1983;22:536-9.

8. Lang EK, Hanano A, rudman E. Pyeloduodenal fistula diagnosed on parenchymal excretory phase computerized tomography. J Urol 2010;184:334.

9. Lee KN, Hwang IH, Shin MJ, Lee SB, Kim IY, Lee DW, et al. Pyeloduodenal fistula successfully treated by endoscopic ligation without surgical nephrectomy: case report. J Korean Med Sci 2014;29:141-4.

10. Herbert FB, Goodacre B, Neal DE Jr. Successful conservative management of nephrocolic fistula. J Endourol 2001;15:281-3.

11. Desmond JM, Evans SE, Couch A, Morewood DJ. Pyeloduodenal fistulae: a report of two cases and review of the literature. Clin Radiol 1989;40:267-70.

12. Herbert FB, Goodacre B, Neal DE Jr. Successful conservative management of nephrocolic fistula. J Endourol 2001;15: 281-3. 